\title{
Phosphorylation-dependent regulation of Notch1 signaling: the fulcrum of Notch1 signaling
}

\author{
Hye-Jin Lee, Mi-Yeon Kim \& Hee-Sae Park ${ }^{*}$ \\ Hormone Research Center, School of Biological Sciences and Technology, Chonnam National University, Gwangju 61186, Korea
}

\begin{abstract}
Notch signaling plays a pivotal role in cell fate determination, cellular development, cellular self-renewal, tumor progression, and has been linked to developmental disorders and carcinogenesis. Notch1 is activated through interactions with the ligands of neighboring cells, and acts as a transcriptional activator in the nucleus. The Notch1 intracellular domain (Notch1-IC) regulates the expression of target genes related to tumor development and progression. The Notch1 protein undergoes modification after translation by posttranslational modification enzymes. Phosphorylation modification is critical for enzymatic activation, complex formation, degradation, and subcellular localization. According to the nuclear cycle, Notch1-IC is degraded by E3 ligase, FBW7 in the nucleus via phosphorylation-dependent degradation. Here, we summarize the Notch signaling pathway, and resolve to understand the role of phosphorylation in the regulation of Notch signaling as well as to understand its relation to cancer. [BMB Reports 2015; 48(8): 431-437]
\end{abstract}

\section{INTRODUCTION}

Notch signaling is a highly conserved process and plays an important role in the regulation of cellular growth, cell cycle arrest, and cellular development $(1,2)$. In mammals, there are four Notch receptors (Notch1-4) and the abnormal regulation of Notch1 signaling can promote cancer and other disorders $(3,4)$. Notch1 regulates the expression of target genes such as Hes1, Hes5, and Hey1, for the acceleration of cell growth (5). Notch1 is a single transmembrane receptor, which perceives signal transduction mediated by ligands (6). After Notch1 is activated by docking ligands, sequential cleavage occurs to produce Notch1 intracellular domain (Notch1-IC). Notch1-IC enters the nucleus and promotes the displacement of repressive complex which promotes the activation.

${ }^{*}$ Corresponding author. Tel: +82-62-530-0021; Fax: +82-62-5302199; E-mail: proteome@jnu.ac.kr

http://dx.doi.org/10.5483/BMBRep.2015.48.8.107

Received 2 June 2015

Keywords: Cancer, Degradation, Kinase, Notch1, Phosphorylation
Proteins are regulated by post translational modifications (PTMs), which alter the stability and activities of the proteins. PTMs are not template-based since they differ between single and multiple combinations to promote the functions of proteins in a time- and signal-dependent manner. Proteins are subjected to various PTMs, including phosphorylation, acetylation, methylation, hydroxylation and ubiquitination. Phosphorylation is the addition of a phosphate group, taken from ATP, to serine, threonine, or tyrosine, in order to control enzymatic activity and protein-protein interactions. Acetylation is the transfer of an acetyl group from acetyl coenzyme A to a lysine residue, which regulates the protein stability and function. Methylation is the transfer of a methyl group to the lysine or arginine residue of a protein, previously shown to regulate the epigenetic control of gene silencing and activation; however, protein methylation has also been shown to regulate non-histone protein expression to control gene regulation and protein stability. Hydroxylation is the modification of a hydroxyl group to a proline residue under hypoxic conditions in order to adapt to the microenvironment. Ubiquitination is the addition of a ubiquitin conjugate to a lysine residue to regulate cellular location and protein stability. PTMs are reversibly and competitively regulated to control the signaling cascades responsible for the maintenance of cellular homeostasis.

Particularly, protein phosphorylation controls the activation of signaling cascades in response to various stimuli, regulating cell growth and survival. The phosphorylation of Notch1-IC interrupts the formation of the Notch1-IC-induced transactivation complex and promotes ubiquitin-dependent modification targeted by the phosphorylation signal $(7,8)$. Various kinases are known to phosphorylate Notch1, and the expression of these kinases is different in cancers. In addition, Notch1 is mutated to mimic the phosphorylation-deficient form in order to avoid degradation in cancer. Here, we provide an overview of the Notch signaling pathway, and resolve to understand the role of phosphorylation in the regulation of Notch1 signaling.

\section{NOTCH: RECEPTORS AND LIGANDS}

Notch is a single transmembrane receptor involved in signal transduction as a transcription regulator (9). Notch was first discovered over 100 years ago by Morgan and colleagues, who found that the mutation of Notch genes resulted in a de-

ISSN: 1976-670X (electronic edition)

Copyright (C) 2015 by the The Korean Society for Biochemistry and Molecular Biology

(c) This is an open-access article distributed under the terms of the Creative Commons Attribution Non-Commercial License (http://creativecommons.org/licenses/by-nc/4.0) which permits unrestricted non-commercial use, distribution, and reproduction in any medium, provided the original work is properly cited. 
fect of wings in the fruit fly, Drosophila melanogaster. These flies have two ligands, Delta and Serrate, which induce the Notch signal of neighboring cells. Caenorhabditis-elegans has two receptors (Lin-12 and Glp-1) and four ligands (APX-1, LAG-2, ARG-1 and DSL-1). In mammals, there are four Notch receptors (Notch1-4) and five ligand genes (Delta-like 1, 3, 4 and Jagged1, 2). Notch ligands have a Delta/Serrate/Lag-2 (DSL) domain which allows the Notch receptor to identify the ligand and to receive signaling (Fig. 1) (4). Notch consists of various domains that precisely regulate the function. The Notch receptor has 36-epidermal growth factor (EGF)-like repeats essential for the binding of ligands. There are three juxtamembrane repeats subjected to proteolysis in the processing of Notch. Also, Notch has ankyrin repeats, a transactivation domain (TAD) involved in the transactivation of Notch, and a proline, glutamic acid, serine, and threonine (PEST)-degradation domain critical for the short half-life of Notch1 (10).

\section{THE CANONICAL NOTCH1 SIGNALING PATHWAY}

Notch1 is initially produced as a $300 \mathrm{kDa}$ monomer, which exists as a heterodimer at the cell surface prior to signaling. Before Notch1 is transported from the ER to the Golgi apparatus, proper glycosylation modifies the EGF repeats that have a consensus motif for glycosylation (11). Glycosylation-deficient Notch1 is non-functional and cannot be transported to the Golgi apparatus. During the maturation of Notch1 in the trans-Golgi, cleavage occurs by a furin-like convertase (S1 cleavage) for the transportation to the cell membrane (12). This cleavage separates Notch1 into two fragments, making a heterodimer. They move to the membrane and form the trans- membrane protein complex. Then, cell-to-cell communication and proteolytic processing occurs when the extracellular domain of Notch1 is docked onto the DSL domain of a ligand. Secondary hydrolysis is promoted by ligand binding, which cleaves the extracellular domain of the Notch1 receptor (13, 14). The extracellular domain is subjected to secondary hydrolysis by ADAM (A Desintegrin And Metalloproteinase) protease and gamma secretase then cleaves the inner fragment, resulting in the release of the signaling fragment, Notch1 intracellular domain (Notch1-IC) (15). Then, Notch1-IC trans-locates into the nucleus and interacts with CSL ( $\underline{C B F} /$ recombining binding protein suppressor of Hairless [RBP-J $\mathrm{k}]$ in mammals, SuH [Suppressor of Hairless] in Drosophila melanogaster and Lag-1 in C. elegans) and MAML (mastermind-like) and functions as a transcriptional activator to regulate its target gene $(16,17)$.

\section{NUCLEAR CYCLE WITH THE NOTCH1 INTRACELLULAR DOMAIN}

Without Notch1 activation, RBP-J $\kappa$ suppresses the transcription of target genes by forming a complex with co-repressors (18, 19). When Notch1 is activated by ligands, nuclear-translocated Notch1-IC interacts with RBP-Jא through the RBP-J associated molecule (RAM) domain and dissociates the corepressor complex. In addition, Notch1-IC recruits the general coactivator, p300/CBP/Mastermind (MAML-1), and another histone-modifying enzyme $(17,20,21)$. This complex leads to the transcriptional activation of target genes, including members of the Hairy/enhancer-of-split (HES) family, the Hairy/enhancer-of-split related with YRPW motif (Hey) family, nuclear

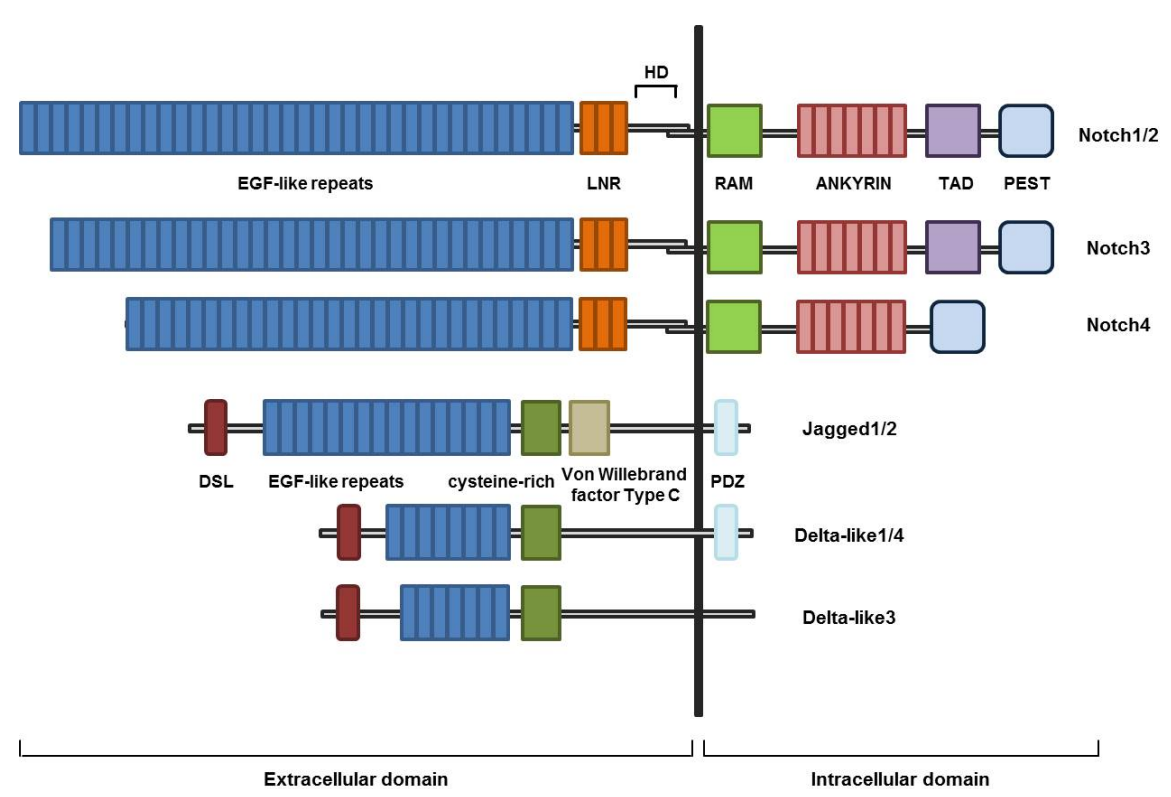

Fig. 1. The molecular structure of the Notch receptor and ligand in mammals. The Notch receptor consists of Notch1-4, and is a heterodimer complex spanning the plasma membrane. Notch has a functional domain, which regulates cellular process. Members of the Delta-like (1, 3 and 4$)$ and Jagged $(1,2)$ families serve as ligands for signal transduction using the DSL residue. The ligands have a cysteine domain and an EGF-like repeat to specifically bind to the Notch receptor and the Von Willebrand factor type C domain for ligand dimerization. 
factor-kappa B (NF-кB), the vascular endothelial growth factor receptor (VEGF), cyclin D1, c-Myc, p21, p27, Akt, etc. (22). In mammals especially, the best-described Notch1 target genes are the transcription factors Hes1, Hes5, and Hey1, the roles of all of which have been well demonstrated in tumor development and progression (23). However, Notch1-induced transactivation is terminated by the phosphorylation of Notch1-IC. The phosphorylation begins with mastermind and the ski-interacting protein (SKIP), which recruits kinases to the TAD or PEST domain (24). Then, the FBW7/ SEL-10 E3 ligases identify the phosphorylation of the PEST domain to promote ubiquitin-mediated degradation. After Notch1-IC is degraded by the Notch1-targeted turn over, the repressors form a complex and inhibit the transcriptional activity of Notch1 target genes (Fig. 2) (25)

\section{PHOSPHORYLATION-DEPENDENT REGULATION OF THE NOTCH1 INTRACELLULAR DOMAIN}

To balance the threshold of Notch1 activity, it is effective and economic to down-regulate protein stability. Various reports have suggested that Notch1 is regulated by posttranslational modification such as phosphorylation and ubiquitination, during the multiple steps of signal transduction (6). The ubiquitination of Notch ligands is necessary to activate the Notch1 signaling pathway. Mind bomb ubiquitinates Delta and induces endocytosis and signal transduction in the signal-receiving cell (26). A Delta mutant that does not have the residue for ubiquitination will fail to induce signal transduction of the Notch1 receptor, while Neuralized (Neur) promotes the endocytosis and degradation of Delta (27). FBW7 recruits the components of an SCF ubiquitin ligase complex through the F-box protein, and recognizes a phosphor-epitope, CPD (Cdc4 phosphodegron; a short linear motif activated by the addition of one or more phosphate groups). Within these substrates via the WD40 domain, ubiquitin-mediated degradation is induced by the proteasome $(28,29)$. Three isoforms, FBW7 $\alpha, \beta$, and $\gamma$, are distributed in the nucleoplasm, cytoplasm, and nucleolus, respectively, after alternative splicing (30). In particular, phosphorylation of the PEST domain is a substrate for recognition by FBW7 in the Notch1 pathway. Mastermind promotes the recruitment of the Cyclin C-CDK8 complex and the hyperphosphorylation of the PEST domain (31). Recently, Cyclin C has been shown to enhance the activity of CDK3 and CDK19,

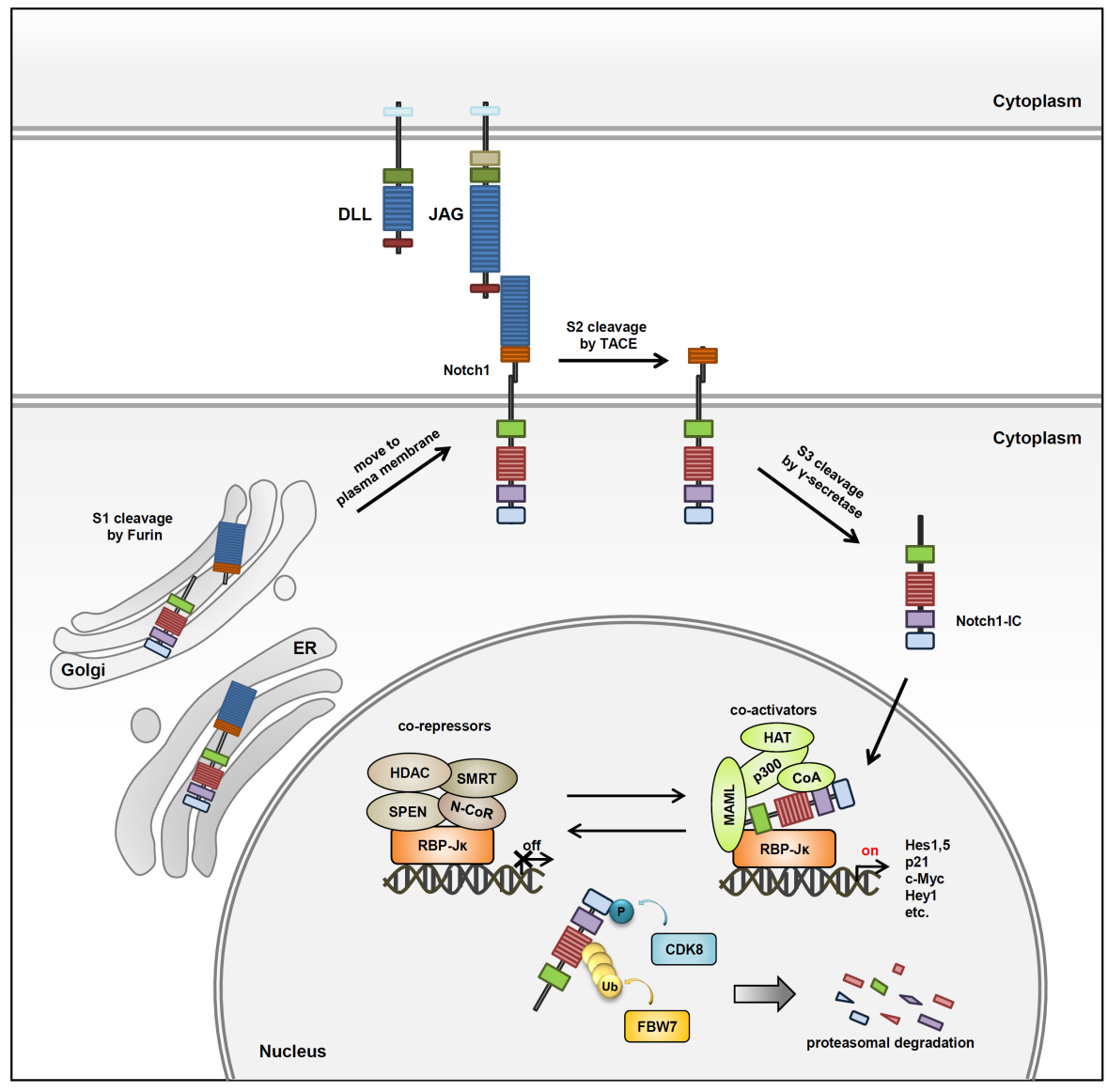

http://bmbreports.org
Fig. 2. The processing of the Notch signaling pathway and the nuclear cycle. Notch1-IC is produced by sequential cleavage via ligand binding. Notch1-IC enters into nucleus and promotes the displacement of a repressive complex and the transactivation of Notch target genes. Notch1-IC activation is regulated by phosphorylation and ubiquitination with CDK8 and FBW7, respectively. HDAC: histone deacetylase, SPEN: split-ends, N-CoR: nuclear receptor corepressor, SMRT: silencing mediator for retinoid and thyroid hormone receptors, MAML: Mastermind, HAT: histone acetyltransferase, CoA: co-activator. 
and to phosphorylate the PEST domain of the Notch1-IC of Thr2512, Ser2514 and Ser2517 (32). The GSK3 $\beta$-mediated phosphorylation of Notch1-IC protects from proteolysis by Itch (33-35). An integrin-linked kinase (ILK) is a component of integrin signaling and phosphorylates Ser2173 of mouse Notch1 (Ser2198 in human Notch1) and down-regulates the protein stability of Notch1-IC, thus decreasing transcriptional activity (36). In addition, the phosphorylation of other domains influences transcriptional regulation. The phosphorylation of the ankyrin domain of Notch1 determines the transcriptional activity of the Notch1 target gene (37). The DYRK1A gene can suppress Notch1-induced transactivation, in a kinase-dependent manner, without affecting protein stability (38). Akt promotes hyperphosphorylation and disrupts the translocation of Notch1-IC, resulting in the inhibition of the transcriptional regulation (39). Nemo-like kinase (NLK) phosphorylates the membrane-tethered Notch1 protein, as well as the Notch intracellular domain. NLK-mediated phosphorylation does not interfere with the nuclear localization of Notch1-IC, but decreases the interaction of Mastermind and the association of the Notch active transcription complex (Fig. 3) (40).

\section{EFFECT OF NOTCH SIGNALING ON CANCER}

Several tumors exploit the potential of Notch1 as an oncogene and tumor suppressor. According to COSMIC data, hematopoietic and lymphoid cancers show a high mutation rate of Notch 1 at $72 \%$, and solid tumors in the upper aerodigestive tract, large intestine, lung, skin, stomach, and breast also show aberrant expression of Notch1. Previous studies reported the Notch1 protein stabilized in T-cell acute lymphoblastic leuke- mia (T-ALL) and solid tumors such as breast cancer, murine mammary cancer and lung cancer $(41,42)$. Patients of T-ALL have shown epigenetic mutations, including the translocation of the 3'-region of Notch1 in T-cell receptor beta locus. The Notch1 intracellular domain (Notch1-IC) is over-expressed, resulting in the activation of target genes. The aberrant expression of Notch1-IC induces the accumulation and cell-cycle arrest of bone marrow progenitor cells, resulting in the tumorigenesis of lymphoid cancer (41). The abnormal expression of Notch1 was also shown in breast cancer with high levels of Notch1-IC being expressed in 20 breast cancer tissues and the negative regulator of Notch1 signaling, Numb, being down-regulated in these tissues (43). According to research in Chinese breast cancer patients using reverse transcription polymerase chain reaction (RT-PCR) and immunohistochemistry, the aberrant expression of Notch1 was shown, especially in those with stage 2 lobular carcinoma (44). In another study, the levels of Notch1-IC and p21 in 109 cases of gastric cancer, a major disease in developing countries, was examined using immunohistochemistry (45). The expression of Notch1-IC increased in the more advanced stage cancers, while the expression of p21 was down-regulated in these cases. This negative correlation consequently promotes the invasion and phenotypic characteristics of the tumor.

In addition to a mutation on the hetero-dimerization domain, the main Notch1 active mutation is a frame shift to codon 2515 in the PEST domain, producing insensitivity to FBW7 (46). The numerous cancer-associated mutations within the CPDs of FBW7 substrates disrupt sensitivity to FBW7 degradation (47). Peptidyl-prolyl cis-trans isomerase 1 (Pin1) regulates the isomerization and inactivation of FBW7 and stabilizes

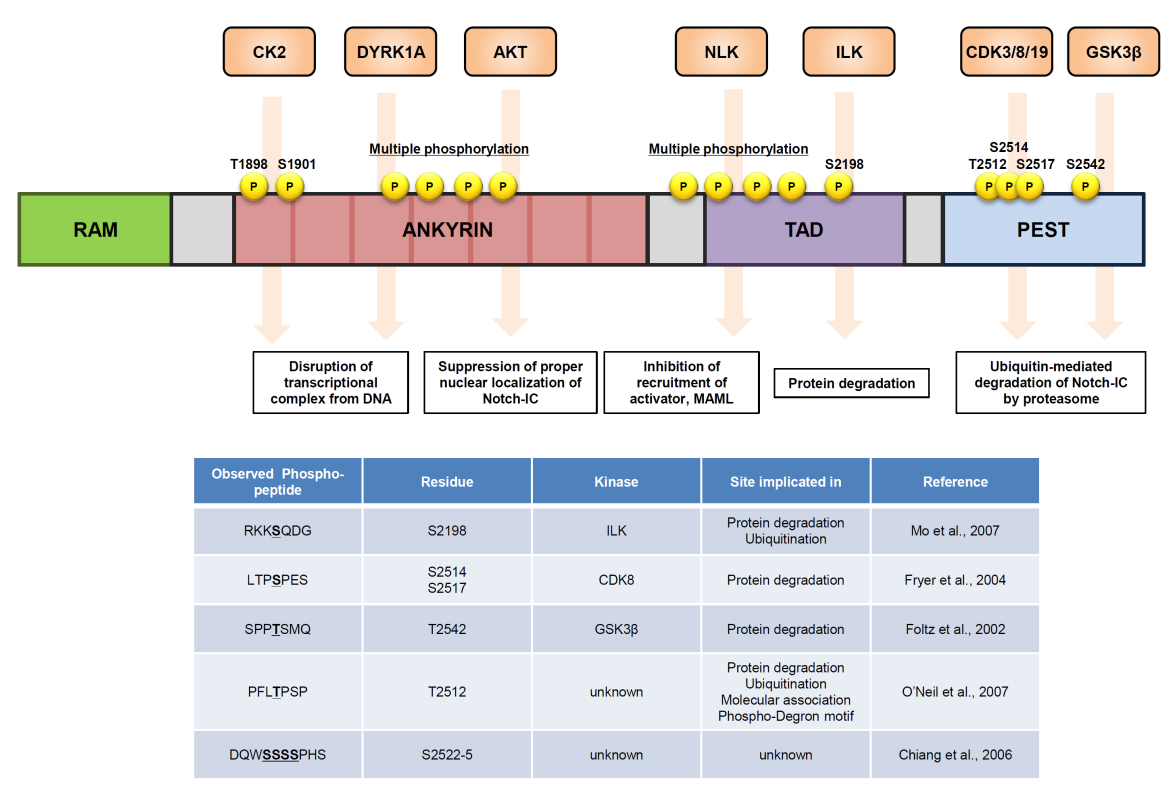

Fig. 3. The domain architecture of mammalian Notch1 receptors and the regulation of Notch-IC by kinases. Notch 1 is a highly conserved transmembrane protein including an ectodomain (EGF-like repeats and LNR domain), a heterodimerization domain (HD), and an intracellular domain (RAM domain, ankyrin repeats, TAD, and PEST domain). Notch1-IC is regulated by the phosphorylation of several kinases, mainly on the ankyrin repeats, to regulate the formation of the transcriptional complex. Furthermore, Notch1-IC phosphorylation of the PEST domain controls the protein stability of Notch1-IC $(59,60)$. 
Notch1-IC, by inhibiting the FBW7-mediated degradation of Notch1-IC (48). Notch1 signaling is engaged in epithelial-mesenchymal transition by regulating the transcription regulators such as Snail, Slug, TGF- $\beta$, FGF, and PDGF and supports the phenotypic and functional changes during tumor progression $(49,50)$. Notch 1 activation promotes the migration and angiogenesis of cancer and increases the self-renewal activity of cancer stem cells (51). Notch1 induces the expression of Sox2, NANOG and Oct4 to support the activity of tumor-initiating cells, cancer stem cells, which are highly related to tumor migration and recurrence (52).

\section{CROSS-TALK BETWEEN THE NOTCH1 SIGNALING PATHWAY AND OTHER SIGNALING PATHWAYS}

Notch1 signaling regulates various factors that are the main regulators of other signaling pathways, including the Pl-3K/Akt, $\mathrm{NF}-\mathrm{\kappa B}, \mathrm{mTOR}$, and TGF- $\beta$ signaling pathways, and interacts with oncogenic proteins (22). A phosphatase, PTEN, is a negative regulator of Akt signaling by dephosphorylation of the active phosphor residue, and is typically mutated in cancer (53). HES1, one of the Notch1 target genes, binds to the promoter region of PTEN and down-regulates the expression, resulting in the stabilization of Akt activity. Furthermore, Akt promotes the protection of cancer cells from apoptosis via the NF-KB signaling pathway, and angiogenesis via the mTOR signaling pathway (54). NF-kB, which plays a role as a transcription factor of the immune system and cell proliferation, activates the expression of Notch1 target genes. Notch1-IC activates NF-kB signaling through direct interaction, and through the RBP-Jא-mediated transcriptional regulation of p100 and p52, which are subunits of NF- $\mathrm{BB}$ (55). In mTOR signaling, mTOR is phosphorylated by the PI-3K/Akt pathway and vice versa to regulate cell growth, differentiation, cell survival and autophagy. Notch1-IC inhibits the expression of the tumor suppressor, p53, which is a key regulator of cell apoptosis, by decreasing functionally active phosphor-residues (Ser15, 20, and 392) via the mTOR and PI-3K/Akt pathways (56). Notch1 signaling is related to growth factors, such as the platelet-derived growth factor (PDGF), HER/ErbB interactions with epithelial growth factors and transforming growth factor- $\alpha$, vascular endothelial growth factor (VEGF), and transforming growth factor (TGF)- $\beta$ (22). TGF- $\beta$ induces Hey 1 , a Notch target gene, and Jagged1, a Notch ligand, to promote epithelial-mesenchymal transition. Notch1-IC forms a transcriptional complex with Smad3, a component of canonical TGF- $\beta$ signaling and regulates the expression of Hes1 by binding to the promoter (57). These signal cross-talks between oncogenic signaling pathways are very sophisticated regulation processes during cell fate determination and cancer development (22).

\section{CONCLUDING REMARKS}

We briefly provided an overview of the Notch1 signaling path- way, and elucidated the role of phosphorylation in the regulation of cancer progression and development. Turnover of Notch1-IC expression was shown to be a key determinant in the regulation of this activity. Several kinases were shown to promote the phosphorylation of Notch1 in the ankyrin domain and PEST domains, which affects the transactivation and protein stability of Notch1. In particular, the phosphodegron, recognized by the E3 ubiquitin ligase FBW7 for degradation, exists in the PEST domain. Additionally, cancer cells were shown to avoid the degradation of Notch1-IC by promoting C-terminal truncation. Cyclin $\mathrm{C}$ and its partners CDK8, CDK3, and CDK19 have been suggested to phosphorylate Notch1-IC on the phosphodegron along with its nearby residues. The Cyclin C-CDK complex promotes the degradation of Notch1-IC and is regarded as a tumor suppressor. However, Cyclin and Cyclin dependent kinases (CDKs) are controlled temporally, according to the cell cycle mechanism, and Notch1 functions in cell fate determination and differentiation in steady state (58). Thus, further studies focused on the discovery of other kinases targeting the phosphodegron of Notch1-IC may have great potential in suggesting a target protein for cancer therapy. While cancer therapies such as surgery, irradiation therapy, and chemotherapy are primarily used, inducible kinases may be effective in managing cancer growth and survival by down-regulating Notch signaling. Therefore, further studies focused on discovering a new regulator of Notch signaling should be carried out to improve the treatment and control of cancer in humans.

\section{ACKNOWLEDGEMENTS}

This research was supported by a grant from the Korea Health Technology R\&D Project through the Korea Health Industry Development Institute (KHIDI), funded by the Ministry of Health \& Welfare, Republic of Korea (grant number A121274).

This research was also supported by the Basic Science Research Program through the National Research Foundation of Korea (NRF), funded by the Ministry of Science, ICT and future Planning (NRF-2014R1A4A1003642).

\section{REFERENCES}

1. Borggrefe $T$ and Oswald F (2009) The Notch signaling pathway: transcriptional regulation at Notch target genes. Cell Mol Life Sci 66, 1631-1646

2. Artavanis-Tsakonas S, Rand MD and Lake RJ (1999) Notch signaling: cell fate control and signal integration in development. Science 284, 770-776

3. Bolos V, Grego-Bessa J and de la Pompa JL (2007) Notch signaling in development and cancer. Endocr Rev 28, 339-363

4. Cordle J, Johnson S, Tay JZ et al (2008) A conserved face of the Jagged/Serrate DSL domain is involved in Notch trans-activation and cis-inhibition. Nat Struct Mol Biol 15, 849-857

5. Allenspach EJ, Maillard I, Aster JC and Pear WS (2002) 
Notch signaling in cancer. Cancer Biol Ther 1, 466-476

6. Andersson ER, Sandberg R and Lendahl U (2011) Notch signaling: simplicity in design, versatility in function. Development 138, 3593-3612

7. Lai EC (2002) Keeping a good pathway down: transcriptional repression of Notch pathway target genes by CSL proteins. EMBO Rep 3, 840-845

8. Foltz DR and Nye JS (2001) Hyperphosphorylation and association with RBP of the intracellular domain of Notch1. Biochem Biophys Res Commun 286, 484-492

9. Bray SJ (2006) Notch signalling: a simple pathway becomes complex. Nat Rev Mol Cell Biol 7, 678-689

10. Fiuza UM and Arias AM (2007) Cell and molecular biology of Notch. J Endocrinol 194, 459-474

11. Jafar-Nejad H, Leonardi J and Fernandez-Valdivia R (2010) Role of glycans and glycosyltransferases in the regulation of Notch signaling. Glycobiology 20, 931-949

12. Logeat F, Bessia C, Brou C et al (1998) The Notch1 receptor is cleaved constitutively by a furin-like convertase. Proc Natl Acad Sci U S A 95, 8108-8112

13. Chillakuri CR, Sheppard D, Lea SM and Handford PA (2012) Notch receptor-ligand binding and activation: insights from molecular studies. Semin Cell Dev Biol 23, 421-428

14. Mumm JS, Schroeter EH, Saxena MT et al (2000) A ligand-induced extracellular cleavage regulates gamma-secretase-like proteolytic activation of Notch1. Mol Cell 5, 197-206

15. Schroeter EH, Kisslinger JA and Kopan R (1998) Notch-1 signalling requires ligand-induced proteolytic release of intracellular domain. Nature 393, 382-386

16. Krejci A and Bray S (2007) Notch activation stimulates transient and selective binding of $\mathrm{Su}(\mathrm{H}) / \mathrm{CSL}$ to target enhancers. Genes Dev 21, 1322-1327

17. McElhinny AS, Li JL and Wu L (2008) Mastermind-like transcriptional co-activators: emerging roles in regulating cross talk among multiple signaling pathways. Oncogene 27, 5138-5147

18. Oswald F, Winkler M, Cao Y et al (2005) RBP-Jkappa/ SHARP recruits CtIP/CtBP corepressors to silence Notch target genes. Mol Cell Biol 25, 10379-10390

19. Brou C, Logeat F, Lecourtois M et al (1994) Inhibition of the DNA-binding activity of Drosophila suppressor of hairless and of its human homolog, KBF2/RBP-J kappa, by direct protein-protein interaction with Drosophila hairless. Genes Dev 8, 2491-2503

20. Wallberg $A E$, Pedersen $K$, Lendahl $U$ and Roeder RG (2002) p300 and PCAF act cooperatively to mediate transcriptional activation from chromatin templates by notch intracellular domains in vitro. Mol Cell Biol 22, 7812-7819

21. Fryer CJ, Lamar E, Turbachova I, Kintner $C$ and Jones KA (2002) Mastermind mediates chromatin-specific transcription and turnover of the Notch enhancer complex. Genes Dev 16, 1397-1411

22. Guo S, Liu M and Gonzalez-Perez RR (2011) Role of Notch and its oncogenic signaling crosstalk in breast cancer. Biochim Biophys Acta 1815, 197-213

23. Yatim A, Benne C, Sobhian B et al (2012) NOTCH1 nuclear interactome reveals key regulators of its transcrip- tional activity and oncogenic function. Mol Cell 48, 445-458

24. Zhou S, Fujimuro M, Hsieh JJ et al (2000) SKIP, a CBF1-associated protein, interacts with the ankyrin repeat domain of NotchlC To facilitate NotchIC function. Mol Cell Biol 20, 2400-2410

25. Wu G, Lyapina S, Das I et al (2001) SEL-10 is an inhibitor of notch signaling that targets notch for ubiquitin-mediated protein degradation. Mol Cell Biol 21, 7403-7415

26. Itoh M, Kim CH, Palardy G et al (2003) Mind bomb is a ubiquitin ligase that is essential for efficient activation of Notch signaling by Delta. Dev Cell 4, 67-82

27. Lai EC, Deblandre GA, Kintner C and Rubin GM (2001) Drosophila neuralized is a ubiquitin ligase that promotes the internalization and degradation of delta. Dev Cell 1, 783-794

28. Minella AC and Clurman BE (2005) Mechanisms of tumor suppression by the SCF(Fbw7). Cell Cycle 4, 1356-1359

29. Nash P, Tang X, Orlicky S et al (2001) Multisite phosphorylation of a CDK inhibitor sets a threshold for the onset of DNA replication. Nature 414, 514-521

30. Welcker M and Clurman BE (2008) FBW7 ubiquitin ligase: a tumour suppressor at the crossroads of cell division, growth and differentiation. Nat Rev Cancer 8, 83-93

31. Fryer CJ, White JB and Jones KA (2004) Mastermind recruits CycC:CDK8 to phosphorylate the Notch ICD and coordinate activation with turnover. Mol Cell 16, 509-520

32. Li N, Fassl A, Chick J et al (2014) Cyclin C is a haploinsufficient tumour suppressor. Nat Cell Biol 16, 1080-1091

33. Espinosa L, Ingles-Esteve J, Aguilera C and Bigas A (2003) Phosphorylation by glycogen synthase kinase-3 beta down-regulates Notch activity, a link for Notch and Wnt pathways. J Biol Chem 278, 32227-32235

34. Jin $\mathrm{YH}$, Kim H, Oh M, Ki H and Kim K (2009) Regulation of Notch1/NICD and Hes1 expressions by GSK-3alpha/beta. Mol Cells 27, 15-19

35. Foltz DR, Santiago MC, Berechid BE and Nye JS (2002) Glycogen synthase kinase-3beta modulates notch signaling and stability. Curr Biol 12, 1006-1011

36. Mo JS, Kim MY, Han SO et al (2007) Integrin-linked kinase controls Notch1 signaling by down-regulation of protein stability through Fbw7 ubiquitin ligase. Mol Cell Biol $27,5565-5574$

37. Ranganathan P, Vasquez-Del Carpio R, Kaplan FM et al (2011) Hierarchical phosphorylation within the ankyrin repeat domain defines a phosphoregulatory loop that regulates Notch transcriptional activity. J Biol Chem 286, 28844-28857

38. Fernandez-Martinez J, Vela EM, Tora-Ponsioen M, Ocana $\mathrm{OH}$, Nieto MA and Galceran J (2009) Attenuation of Notch signalling by the Down-syndrome-associated kinase DYRK1A. J Cell Sci 122, 1574-1583

39. Song J, Park S, Kim M and Shin I (2008) Down-regulation of Notch-dependent transcription by Akt in vitro. FEBS Lett 582, 1693-1699

40. Ishitani T, Hirao T, Suzuki M et al (2010) Nemo-like kinase suppresses Notch signalling by interfering with formation of the Notch active transcriptional complex. Nat Cell Biol 12, 278-285 
41. Weng AP, Ferrando AA, Lee $\mathrm{W}$ et al (2004) Activating mutations of $\mathrm{NOTCH} 1$ in human $\mathrm{T}$ cell acute lymphoblastic leukemia. Science 306, 269-271

42. Ranganathan $P$, Weaver KL and Capobianco AJ (2011) Notch signalling in solid tumours: a little bit of everything but not all the time. Nat Rev Cancer 11, 338-351

43. Stylianou S, Clarke RB and Brennan K (2006) Aberrant activation of notch signaling in human breast cancer. Cancer Res 66, 1517-1525

44. Ma D, Dong X, Zang S et al (2011) Aberrant expression and clinical correlation of Notch signaling molecules in breast cancer of Chinese population. Asia Pac J Clin Oncol 7, 385-391

45. Luo DH, Zhou Q, Hu SK et al (2014) Differential expression of Notch1 intracellular domain and p21 proteins, and their clinical significance in gastric cancer. Oncol Lett 7, 471-478

46. Grabher C, von Boehmer H and Look AT (2006) Notch 1 activation in the molecular pathogenesis of T-cell acute lymphoblastic leukaemia. Nat Rev Cancer 6, 347-359

47. Erbilgin Y, Sayitoglu M, Hatirnaz O et al (2010) Prognostic significance of $\mathrm{NOTCH} 1$ and FBXW7 mutations in pediatric T-ALL. Dis Markers 28, 353-360

48. Min SH, Lau AW, Lee TH et al (2012) Negative regulation of the stability and tumor suppressor function of Fbw7 by the Pin1 prolyl isomerase. Mol Cell 46, 771-783

49. Sahlgren C, Gustafsson MV, Jin S, Poellinger L and Lendahl U (2008) Notch signaling mediates hypoxia-induced tumor cell migration and invasion. Proc Natl Acad Sci U S A 105, 6392-6397

50. Espinoza I and Miele L (2013) Deadly crosstalk: Notch signaling at the intersection of EMT and cancer stem cells. Cancer Lett 341, 41-45

51. Marignol L, Rivera-Figueroa K, Lynch T and Hollywood D (2013) Hypoxia, notch signalling, and prostate cancer.
Nat Rev Urol 10, 405-413

52. Kong D, Banerjee S, Ahmad A et al (2010) Epithelial to mesenchymal transition is mechanistically linked with stem cell signatures in prostate cancer cells. PLoS One 5, e12445

53. Palomero T, Sulis ML, Cortina M et al (2007) Mutational loss of PTEN induces resistance to NOTCH1 inhibition in T-cell leukemia. Nat Med 13, 1203-1210

54. Bedogni B, Warneke JA, Nickoloff BJ, Giaccia AJ and Powell MB (2008) Notch1 is an effector of Akt and hypoxia in melanoma development. J Clin Invest 118, 3660-3670

55. Oswald F, Liptay S, Adler G and Schmid RM (1998) NF-kappaB2 is a putative target gene of activated Notch-1 via RBP-Jkappa. Mol Cell Biol 18, 2077-2088

56. Mungamuri SK, Yang X, Thor AD and Somasundaram K (2006) Survival signaling by Notch1: mammalian target of rapamycin (mTOR)-dependent inhibition of p53. Cancer Res 66, 4715-4724

57. Zavadil J, Cermak L, Soto-Nieves $\mathrm{N}$ and Bottinger EP (2004) Integration of TGF-beta/Smad and Jagged1/Notch signalling in epithelial-to-mesenchymal transition. EMBO J 23, 1155-1165

58. Holt LJ (2012) Regulatory modules: Coupling protein stability to phopshoregulation during cell division. FEBS Lett 586, 2773-2777

59. O'Neil J, Grim J, Strack P et al (2007) FBW7 mutations in leukemic cells mediate NOTCH pathway activation and resistance to gamma-secretase inhibitors. J Exp Med 204, 1813-1824

60. Chiang MY, Xu ML, Histen G et al (2006) Identification of a conserved negative regulatory sequence that influences the leukemogenic activity of NOTCH1. Mol Cell Biol 26, 6261-6271 